\title{
Cronología y periodización del megalitismo en Galicia y norte de Portugal
}

\author{
RAMÓN FÁBREgAS VALCARCE
}

\section{INTRODUCCIÓN}

A despecho de la importancia cuantitativa que poseen los monumentos de la provincia megalítica noroccidental de la Península lbérica, la investigación sobre este tipo de manifestaciones ha adolecido de un cierto retraso en relación con la dinámica desarrollada en otras áreas de España y Portugal. Ciertamente, con las consabidas excepciones, el número de actuaciones arqueológicas llevadas a cabo en el NW era mínimo y ello incidia gravemente en el conocimiento y perspectivas que sobre el mundo megalítico podían tenerse. Significativamente, las periodizaciones propuestas se basaban o bien en dataciones realizadas en otras regiones, o bien en consideraciones evolutivas en el orden arquitectónico o de la cultura material que con frecuencia pecaban de apriorismo o unilinearidad. Esta tónica, un tanto deprimente, ha dado un vuelco importante en los últimos años, particularmente desde los inicios de la década de los ochenta, debido a los trabajos llevados a cabo en el núcleo megalítico de Serra da Aboboreira, hoy en día el mejor datado de toda la Península, así como a la intensificación en el número de excavaciones realizadas con una metodología científica en Galicia. En consecuencia, hoy se dipone de un elenco de dataciones que permiten, con un cierto margen de provisionalidad, encuadrar las construcciones tumulares en un marco temporal definido y formular algunas hipótesis en cuanto a su periodización. 
Existen túmulos que carecen de cámara ortostática por razones distintas del habitual saqueo. Su inclusión de este trabajo viene determinada por la conciencia de que dentro de la órbita megalítica ha existido un abanico de soluciones, abarcando monumentos carentes de construcción pétrea interna, que sin embargo pueden eventualmente presentar una cronología y una ubicación parejas a las de los megalitos comme il faut.

\section{PROPUESTAS DE PERIODIZACIÓN}

En un trabajo publicado póstumamente (1973: 115), López Cuevillas postuló una división del fenómeno megalítico gallego en dos períodos sucesivos: el primero se caracterizaria por la construcción de túmulos conteniendo cámaras poligonales cerradas 0 , en ocasiones, dotadas de un corredor poco desarrollado. Una segunda fase vendría definida por cistas, túmulos sin cámara y sepulturas planas albergando cerámicas campaniformes o de tipo Penha, puntas de flecha de base recta o cóncava, hachas alargadas y de sección circular y mazas, hachas de combate y puñales, éstos últimos llegados del Norte de Europa. Por el contrario, en el interior de los monumentos del primer período se encontrarian artefactos de tradición indigena y arcaizante.

Posteriormente otros investigadores han abundado en el modelo anterior, modificándolo en un sentido tripartito. Rodríguez Casal (1979: 109) propone una evolución en tres fases: una primera, caracterizada por túmulos con dólmenes simples, abiertos o cerrados y cámaras con corredor corto en cuyo interior se encuentran hachas de sección oval, microlitos geométricos y láminas de sílex, junto con cerámicas lisas. Un segundo momento vendría dado por sepulturas de corredor más desarrollado (aunque sin alcanzar las dimensiones de construcciones análogas en otras regiones de la Península), en los que aparecen como artefactos caracteristicos las puntas de flecha de base triangular y hachas con diferentes tipos de sección. Finalmente, un tercer período en el que se encuentran cámaras de dimensiones reducidas, "cistoides", conteniendo hachas de sección aplanada, mazas y otros útiles con perforación para el enmangue. A grandes rasgos, este esquema ha sido mantenido por otros investigadores gallegos (VV.AA. 1979: 26; Vázquez 1979: 24; Rodriguez Casal 1983: 219) con leves modificaciones, como la introducción de nuevas variables que tratan de describir las diferentes opciones escogidas para 
expresar la relación túmulo/entorno o la dialéctica cámara/túmulo (Fábregas y Criado 1986).

En el Norte de Portugal, las excavaciones realizadas en los últimos diez años, asi como los trabajos realizados anteriormente sobre el megalitismo en áreas próximas (Beira Alta), para las que se disponía de dataciones radiocarbónicas desde hace más tiempo, han llevado a los especialistas del país vecino a propugnar un modelo que se aleja de las tesis evolucionistas unilineares, valorando en cambio el polimorfismo de los túmulos excavados en la Serra da Aboboreira que se manifestaría de un modo parcialmente sincrónico, aunque admitiendo la posibilidad de un cambio desde formas arquitectónicas simples a otras más complejas y de mayor tamaño (V. Jorge 1983-84: 44; 1986; 1988:6).

\section{ANÁLISIS DE LAS DATACIONES}

Independientemente del rigor y fiabilidad con que se lleve a cabo el análisis de las muestras por parte de los correspondiente laboratorios, existe una serie de variables que deben ser tenidas en cuenta a la hora de valorar en su exacta medida las dataciones radiocarbónicas.

Un total de seis monumentos (37,5 por 100 del conjunto considerado) disponen de tan sólo una fecha absoluta, lo cual nos obliga a mantener una prudente reserva a la hora de utilizar esos datos, ya que no se debe descartar la posibilidad de que contaminaciones o remociones puedan alterar el resultado, como se puede comprobar si se revisan aquellos túmulos para los que se poseen varias dataciones. Por otro lado, la procedencia estratigráfica de las muestras analizadas puede inducir una valoración diferente de la cronologia obtenida. El caso más favorable lo constituyen las hogueras halladas bajo los túmulos (11 casos, 68,7 por 100 del total), a veces selladas bajo una capa de tierra aportada por los propios constructores durante la erección de la sepultura. En estas circunstancias se reducen las posibilidades de que los carbones sufran alteraciones con posterioridad a su formación, la cual marcará una cronologia post quem para el túmulo que se encuentra encima, si bien no es factible generalmente estimar el espacio de tiempo que media entre el encendido de las hogueras y la construcción que las ha cubierto ya que en principio no puede descartarse que en algún caso 
aquéllas hayan formado parte de un hábitat bastante anterior ' . Con todo, la frecuencia con que se ha señalado la existencia de restos de fuego bajo los túmulos parece indicar una práctica generalizada por parte de las poblaciones que edifican las sepulturas megalíticas, ya sea por razones funcionales (limpieza de la vegetación) o bien como una parte de ceremonias previas a la construcción. Excepcionalmente, tenemos un monumento (Mina do Simâo) en el que se ha podido datar carbones procedentes de la capa superior del paleosuelo y otros hallados en el nivel inferior de la cámara, en una esquina que había quedado milagrosamente a salvo de los saqueos. Los resultados de dichos análisis radiocarbónicos muestran un desfase prácticamente insignificante entre el momento en que se encendió fuego en el terreno donde luego se iba a levantar el túmulo y la utilización más antigua de la cámara contenida en éste.

Un cierto número de monumentos ( 6 casos, 37,5 por 100 del total) poseen fechas radiocarbónicas que han sido obtenidas a partir de muestras localizadas entre las tierras del túmulo. Éste es uno de los casos más problemáticos puesto que en muchos casos no se puede establecer una correlación efectiva entre la formación de los carbones y la construcción del monumento y de hecho buena parte de las dataciones discutidas tienen esta procedencia. Las alteraciones principales que pueden afectar a los materiales orgánicos objeto del análisis atañen por una parte a los rejuvenecimientos producidos por la acción de las raices penetrando en las capas de tierra que componen el túmulo. En un sentido opuesto, durante las labores de acarreo y extracción de tierras para levantar el túmulo pueden recogerse de forma casual carbones u otros restos orgánicos de cronología anterior. Este tipo de fenómenos podría explicar las sorprendentes diferencias entre las fechas radiocarbónicas de Outeiro de Ante 3, algunas de las cuales, por otra parte (GIF-4857 y GIF-4858), eran considerablemente más antiguas que las comúnmente aceptadas para monumentos de características similares, lo que conjuntamente con las mencionadas diferencias entre las cuatro fechas publicadas, ha hecho que fuesen contempladas con mucha reserva ${ }^{2}$. Una recogida de carbo-

' Esta podría ser la explicación de la fecha, algo más elevada de lo esperado, obtenida a partir de carbones localizados bajo la estructura pétrea que conformaba el túmulo de Outeiro de Gregos 5.

${ }^{2}$ De todos modos el carácter de chronological outliers que parecían mostrar las dataciones más antiguas de este monumentos se ha disipado en parte al darse a conocer las fechas radiocarbónicas de Châ de Parada 4 que dentro de una gran coherencia global remontan el inicio de su construcción hasta mediados del IV..$^{\circ}$ milenio (en fechas no calibradas). 
nes antiguos en el curso de las labores de amontonamiento de tierras durante la construcción del túmulo podría ser la explicación de las disparidades señaladas entre las fechas radiocarbónicas de Outeiro de Gregos 2, por lo que en principio habría que primar el valor suministrado por la muestra procedente del paleosuelo bajo el mismo. La cronología de Os Campiños 6 constituye un ejemplo aparte puesto que si bien lo hemos incluido entre el grupo que posee fechas obtenidas a partir de muestras procedentes entre las tierras del túmulo, se trata del único caso en que se han datado carbones recuperados de una capa de tierra inalterada que sellaba el corredor y aunque se trata de una fecha aislada, resulta coherente respecto al contexto arqueológico y a la cronología obtenida recientemente en Châ de Parada 1.

\section{TÚMULOS SIN CÁMARA}

Châ de Santinhos 2; GIF-6784; $4990 \pm 50$ b.p. (hogar 1 bajo el túmulo); GIF-6785: $4930 \pm 50$ b.p. (hogar 2 bajo el túmulo).

Outeiro de Gregos 5; CSIC-659: $4780 \pm 60$ b.p. (muestra procedente del nivel superior de la capa de tierra bajo el monumento).

SEPULTURAS DE CORREDOR

Châ de Parada 1; ICEN-172; $4900 \pm 260$ b.p.; ICEN-173: $4610 \pm 45$ b.p. (dos fragmentos de una misma muestra de carbón procedente del paleosuelo bajo el túmulo).

Os Campiños 6; GrN-14328: $4300 \pm 60$ b.p. (nivel de tierra sellando el corredor).

As Pereiras; GaK-11821: $4850 \pm 210$ b.p. (bolsada de carbón dentro del túmulo).

\section{SEPULTURAS CON CÁMARA SIMPLE}

Outeiro de Ante 3; GIF-4857: $5780 \pm 80$ b.p.; GIF-4858: $5540 \pm 90$ b.p.; GIF-4856; $4800 \pm 80$ b.p.; GIF-4859: $4090 \pm 120$ b.p. (todas las muestras provienen de hogueras entre las tierras del túmulo).

Outeiro de Gregos 2; KN-2768: $5500 \pm 70$ b.p. (muestra dentro del túmulo); CSIC-547: $4950 \pm 50$ b.p. (muestra procedente del paleosuelo sellado bajo el túmulo).

Meninas do Crasto 2; CSIC-656: $5260 \pm 50$ b.p.; CSIC-658: $5260 \pm 60$ b.p.; CSIC-659: $5260 \pm 60$ b.p. (todas las muestras provienen de la capa superior del paleosuelo sellado bajo el túmulo). 
Châ de Santinhos 1; GIF-6783: $4980 \pm 50$ b.p. (hogar estructurado en el nivel superior del paleosuelo).

Outeiro de Gregos 3; KN-2765: $5200 \pm 65$ b.p. (carbones del interior del túmulo); KN-2766: $5230 \pm 75$ b.p. (carbones del interior del túmulo); KN-2767: $2510 \pm 65$ b.p. (zona violada del interior de la cámara).

Abogalheira 1; KN-2955: $4590 \pm 85$ b.p. (capa de carbones en el interior del túmulo).

Meninas do Crasto 4; GaK-10943: $4220 \pm 140$ b.p.; CSIC-660: $3800 \pm 50$ b.p.; CSIC- 661 : $3830 \pm 50$ b.p. (todas las muestras provienen del nivel superior del paleosuelo sellado bajo el túmulo).

Mina do Simâo; CSIC-717: $5130 \pm 90$ b.p. (capa superior del paleosuelo); CSIC-716: $5050 \pm 70$ b.p.; CSIC-715: $5010 \pm 70$ b.p. (ambas muestras en el nivel inferior intacto de la cámara).

Châ de Parada 4; ICEN-162: $5470 \pm 45$ b.p.; ICEN-170: $5530 \pm 300$ b.p.; ICEN-171: $5730 \pm 130$ b.p. (todas provienen del hogar 1 sobre el nivel del paleosuelo); ICEN-169: $5420 \pm 40$ b.p. (hogar 2 sobre el nivel del paleosuelo).

Chan da Cruz 1; GaK-11395: $5890 \pm 120$ b.p. (hogar sobre un suelo preparado bajo el túmulo); CSIC-642: $5210 \pm 50$ b.p. (hoguera sobre el suelo preparado bajo el túmulo).

As Rozas 1; GaK-11189: $5150 \pm 140$ b.p. (hoguera situada sobre un suelo preparado bajo el túmulo).

Un grupo de dataciones suministradas por el laboratorio de la Universidad de Gakushuin resultan discordantes no sólo con el contexto arqueológico de donde se ha obtenido la muestra ${ }^{3}$, sino también con los resultados obtenidos por otros laboratorios sobre materiales con la misma procedencia estratigráfica. Asi en Chan da Cruz 1 un hogar y una hoguera probablemente contemporáneos dieron unas fechas separadas 680

${ }^{3}$ Generalmente, suponiendo una cronología más elevada de lo esperado. Un ejemplo de ésto lo tenemos en la fecha de Outeiro de Ante 2 (GaK-10937: $5920 \pm 130$ b.p.), abiertamente rechazada por el autor de la excavación por lo que, dado su carácter único, la hemos excluido de nuestro trabajo (ver a este respecto: A. -A. H. B. Gonçalves, Escavaçâo de mamoa n. ${ }^{\circ} 2$ de Outeiro de Ante, Arqueología, 9, 1984, 42; V. O. Jorge, Novas dataçôes de radiocarbono para mamoas do concelho de Baiâo, Arqueología, 11, 1985, 183. 
años entre sí, siendo la proporcionada por Gakushuin la más elevada 4 . Debemos por tanto contemplar con gran prudencia las dataciones de As Pereiras y As Rozas 1, aun cuando éstas puedan ser aparentemente aceptables, dado que no conocemos otras fechas que puedan corroborarlas.

Por último, algunas dataciones con una desviación típica muy amplia (As Pereiras, Châ de Parada 4) dificultan las tareas de comparación, dada la extensión del marco temporal en que puede localizarse la "fecha real" ${ }^{5}$, y sólo se puede salvar en parte ese obstáculo en aquellos casos en los que se dispone de otras dataciones más precisas para el mismo monumento. Si realizamos una media ponderada de las tres fechas obtenidas a partir del hogar 1 de Châ de Parada 4 (ICEN-162, 170 y 171), obtenemos un resultado (5498 \pm 42 b.p.) considerablemente más preciso y que no difiere significativamente del logrado mediante el análisis de los carbones. del hogar 2 del mismo monumento (ICEN-169). Una operación similar llevada a cabo con las fechas de Châ de Parada 1 (obtenidas a partir de dos fragmentos de una misma muestra) privilegiaria la datación ICEN-173.

\section{CONCLUSIONES}

Observando el cuadro con la representacion gráfica de las fechas radiocarbónicas (fig. 1), se advierte un apreciable grado de solapamiento entre ellas que parece sugerir una cierta sincronia entre las diferentes fórmulas de sepultura megalítica. Este aspecto se hace patente asimismo cuando se calibran esas mismas fechas (fig. 2). Sin embargo, dejando al

${ }^{4}$ Hasta el punto de convertir esta sepultura en la más antigua del Noroeste y una de las más antiguas del megalitismo europeo. Queremos agradecer al excavador, R. Patiño, el habernos informado sobre la fecha radiocarbónica dada a conocer por el CSIC.

Algo semejante ocurre con el túmulo de Meninas do Crasto 4 , en el que de nuevo el resultado del análisis de Gakushuin se significa por su mayor antigüedad en relación con las fechas del CSIC.

S A la hora de evaluar las diferencias entre las dataciones radiocarbónicas hemos manejado éstas con un intervalo de confianza correspondiente a 20 , empleado asimismo para corregir dichas fechas. La calibración ha sido realizada según las curvas de Pearson et al. (1986). 


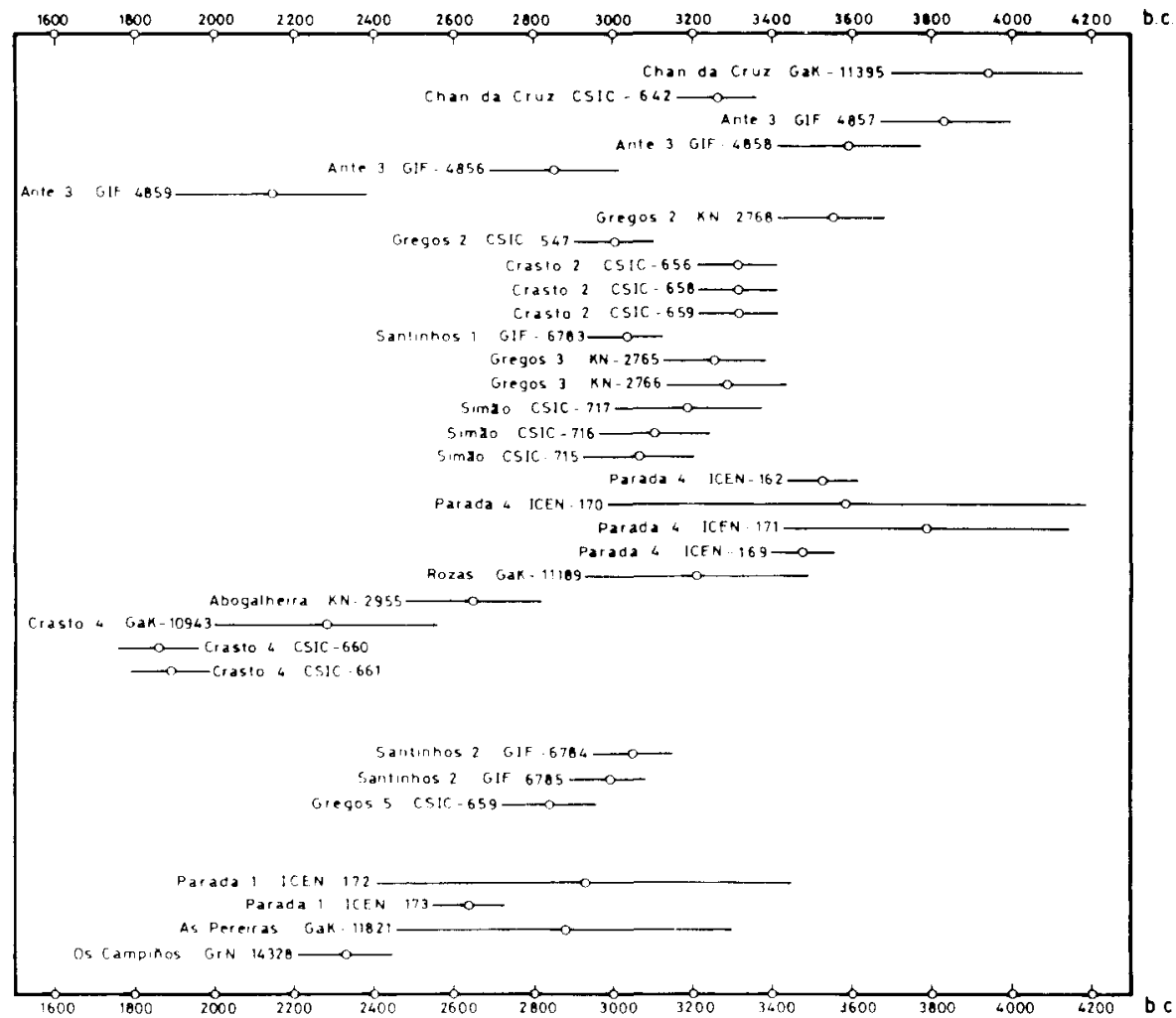

Figura 1. Fechas radiocarbónicas para el megalitismo de Galicia y Norte de Portugal (intervalos de confianza correspondientes a 20). Se ha agrupado las dataciones según los tres grandes tipos arquitectónicos. No está representada la fecha KN-2767 (Gregos 3).

margen algunas dataciones problemáticas, bien por el mismo resultado 0 a causa de las circunstancias de su obtención, algunos hechos comienzan a aflorar.

Primeramente, hay un núcleo de monumentos agrupados en un estrecho margen temporal, en torno al último tercio del $\mathrm{IV}^{\circ}$ milenio (en cronología convencional), constituido por túmulos que albergan una cámara poligonal simple, en unos casos de dimensiones bastante amplias (Mina de Simâo), en otros de proporciones más modestas (Gregos 2 y 3). La posibilidad de remontar la presencia de este tipo de construcciones hasta mediados del IV milenio viene apuntada por la cronología absoluta de Châ de Parada 4 y, con más dudas, por dos de las fechas obtenidas en 


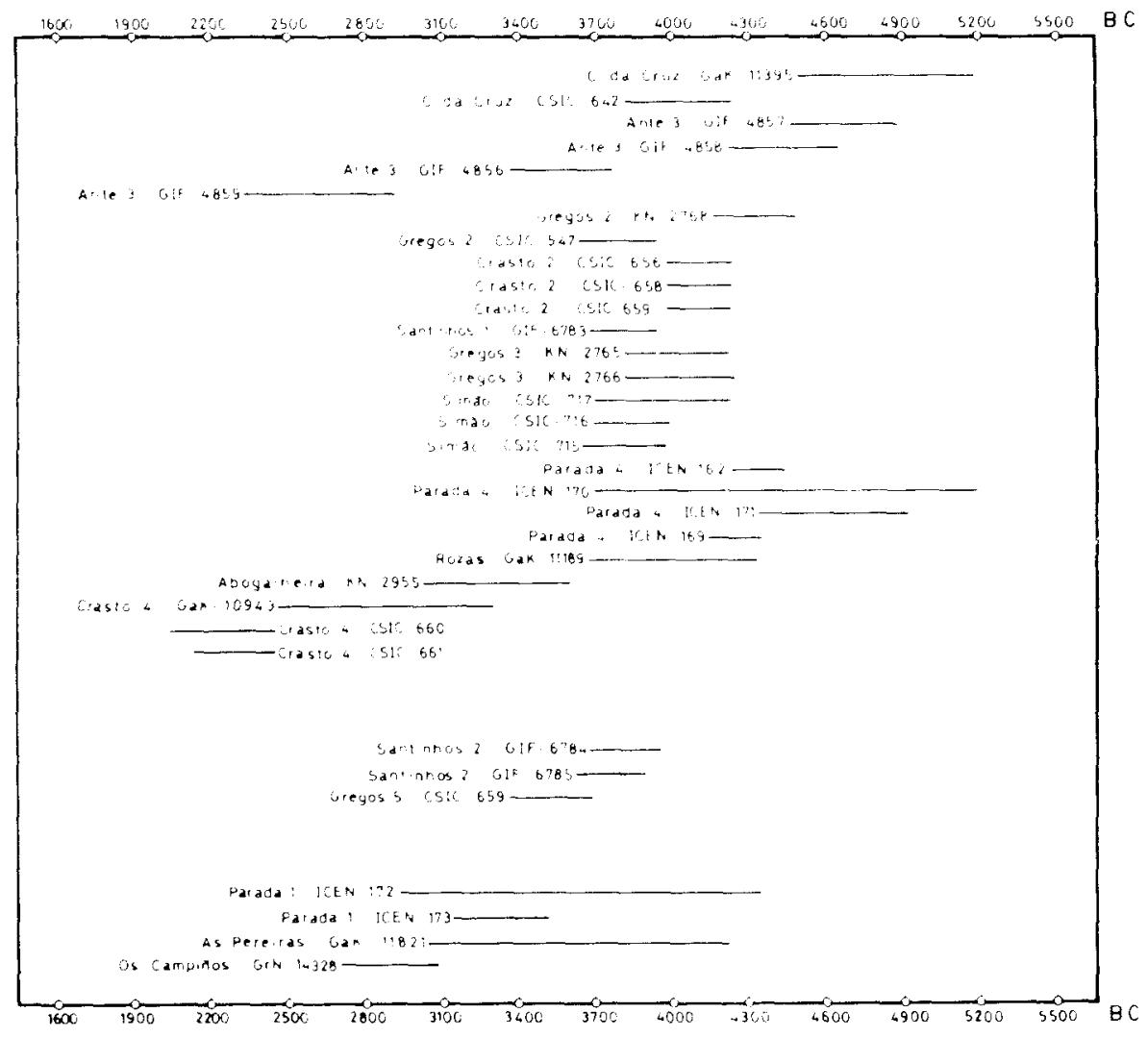

Figura 2. Fechas radiocarbónicas calibradas según la curva de Pearson et al. Se ha representado el margen existente entre los valores máximo y mínimo resultantes de la intersección sobre la curva de calibración de cada datación convencional (con un intervalo de confianza correspondiente a $2 \sigma$ ).

Outeiro de Ante 3. Esta fórmula arquitectónica sigue vigente durante un largo periodo, no sólo en cuanto a reutilización de túmulos construidos con anterioridad, sino también a través de la construcción de dólmenes simples entrado el $11^{\circ}$ milenio (Meninas do Crasto 4), entre los que se ha señalado una tendencia hacia la reducción de tamaño y de sus condiciones de visibilidad dentro del entorno ( $V$. Jorge 1988: 9; Criado, Aira y Díaz 1986: 98, 139; Fábregas y Criado 1986).

Los túmulos sin cámara no acusan aparentemente un gran desfase temporal respecto al núcleo antiguo de sepulcros con cámara simple, siendo significativo el túmulo de Santinhos 2, que se levanta al lado de un dolmen (Santinhos 1), con una cronología absoluta prácticamente idéntica. Es cierto que de nuevo nos encontramos ante una solución 
constructiva con una amplia pervivencia, incluso superior a la acreditada por las sepulturas ortostáticas, ya que en repetidas ocasiones se ha senalado en Galicia la presencia de túmulos carentes de estructura pétrea interna, generalmente de pequeño tamaño y vinculados con cerámica campaniforme (Criado y Vázquez 1982: 58). También en Asturias aparece este tipo de monumentos - si bien de mayores dimensiones-, a los que se atribuye una cronología postmegalítica (Jordá, Domínguez y Aguadé 1972-73) que podria llegar hasta el Bronce Pleno como parece indicar la datación radiocarbónica del túmulo de Piedrafita V (Blas 1985: 134).

Por su parte las sepulturas de corredor, con un menguado número de dataciones todavia, presentan cierta indefinición cronológica: algunas fechas radiocarbónicas apuntan hacia una cierta antigüedad, en los inicios del III milenio (As Pereiras y Parada 1), lo cual sería coherente con la cronología de los sepulcros de corredor de la Beira Alta (Kalb 1987: 105-6) o del Norte de Burgos (Delibes 1984: 102). No obstante creemos, por las razones expuestas en el apartado anterior, que en los ejemplos de Galicia y Norte de Portugal son las fechas más recientes las que presentan mayores garantías, en concreto una de las de Parada 1 (ICEN 173) y la de Os Campiños 6. Estas últimas situarían las sepulturas de corredor, o al menos aquéllas con estructuras de acceso bien desarrolladas, a partir de mediados del III milenio (segunda mitad del IV en fechas calibradas).

En una perspectiva de futuro haría falta realizar excavaciones $-y$ consecutivamente disponer de dataciones absolutas- en túmulos de otras zonas dentro del NW, para verificar, completar o corregir las ideas avanzadas más arriba. Ello es tanto más necesario cuanto hoy en dia las dataciones absolutas disponibles proceden de dos zonas muy delimitadas: el cuadrante costero sudoccidental de Galicia y la Serra da Aboboreira en el Norte de Portugal y nada asegura que las dinámicas de cambio entrevistas en esas áreas sean aplicables en su integridad al conjunto del territorio. En este sentido queda por resolver el problema de la ubicación cronológica de las sepulturas con cámara rectangular, "cistas", sobre las que existe un consenso entre los especialistas para considerarlas tardias, de finales del III milenio, aunque hasta hoy ninguna haya sido objeto de una excavación científica y su datación se haga en función de los materiales (mazas, dobles azuelas, cinceles) hallados en su interior. 


\section{BIBLIOGRAFÍA}

BLAS, M. A., 1985: Piedrafita V. Nuevos aspectos sobre el polimorfismo de las arquitecturas funerarias prehistóricas en el N-N.O. de la Península Ibérica. Arqueología, 12: 129-136.

Criado Boado, F. y Vazouez Varela, J. M., 1982: La cerámica campaniforme en Galicia. Cuadernos do Seminario de Sargadelos, 42, Sada, 104 págs.

Criado Boado, F., Aira Rodríguez, M. J. y Diaz-Fierros Viqueira, F., 1986: La construcción del paisaje: Megalitismo y ecología. Sierra de Barbanza. Arqueoloxía e Investigación, 1, Santiago de Compostela, 177 págs.

DA Cunha, A. M. C. L. y Silva, E. J. L., 1982: Escavaçao da mamoa 1 da Abogalheira. Trabalhos do G.I.A.N., 1: 44 págs.

DELIBES, G., 1984: Fechas de radiocarbono para el megalitismo de la Meseta española. Arqueología, 10: 99-102.

Fabregas Valcarce, R. y Criado Boado, F., 1986: Some aspects of the megalithic culture of NW Iberia. En World Archaeological Congress, The Neolithic of Europe Section, Southampton.

Fabregas Valcarce, R. y Fuente Andres, F., 1984: "Os Campiños" Leiro. Arqueologia 83: 221-222, Madrid.

Gonçalves, A.-A. H. B., 1984: Escavaçao da mamoa n. ${ }^{\circ} 2$ de Outeiro de Ante. Arqueologia, 9: 22-44.

Jordá Cerda, F., Dominguez Garcia, E. y Aguadé, J., 1973-74: Notas sobre los túmulos dolménicos de Campiello (Tineo) y su edad postdolménica. Zephyrus, 33-34: 131-152.

JORGE, S. O., 1985: Datas de Carbono 14 para a pré-historia recente do Norte de Portugal: Os dados e os problemas. Arqueologia, 12: 154183.

JoRGE, V. O., 1979: Escavaçâo das mamoas 2 e 3 de Outeiro de Gregos. Revista de Guimarâes, 89: 251-264. 
- 1980: Escavaçao da mamoa 2 de Outeiro de Gregos. Revista de Guimarâes, 90: 191:210.

- 1980: Escavaçâo da mamoa 3 de Outeiro de Ante. En Seminario de Arqueologia do Noroeste Peninsular, Guimarâes, vol. 1: 41-59.

- 1983-84: Megalitismo do Norte de Portugal: Un novo balanço. En Coloquio Inter-Universitario de Arqueologia do Noroeste, Portugalia 4-5: 37-45.

- 1983: Escavaçâo das mamoas 2 e 4 de Meninas do Crasto. Arqueología, $7:$ :23-43.

- 1983: Uma data de radiocarbono para a mamoa 4 de Meninas do Crasto. Arqueologia, 8: 23.

- 1984: Escavaçâo da mamoa da Mina do Simâo. Arqueologia, 9: 3-21.

- 1985: Les tumulus de Châ de Santinhos. Arqueologia, 12: 98-129.

- 1985: Novas dataçôes de radiocarbono para mamoas do concelho de Baiâo. Arqueologia, 11: 182-183.

- 1985: Uma dataçâo pelo radiocarbono para a mamoa 5 de Outeiro de Gregos (Baiâo). Arqueologia, 12: 94-95.

- 1986: Les monuments mégalithiques du Nord du Portugal. En World Archaeological Congress: The Neolithic of Europe Section, Southampton.

- 1988: Campo arqueológico da Serra da Aboboreira. Arqueología do concelho de Baiâo. Resultados de 10 anos de traballo. Arqueologia, 17: 5-26.

- 1988: Datas de Carbono 14 para a mamoa de Châ de Parada 4. Arqueología, 17: 121-123.

JORGE, V. O. y Alfonso, F., 1987: Datas de Carbono 14 para a mamoa da Mina do Simâo. Arqueologia, 15: 92-93.

JoRGE, V. O. y BETTENCOURT, A., 1988: Sondagens arqueológicas na Mamoa 1 de Châ de Parada. Arqueologia, 17: 73-118.

JoRGE, V. O. y MoreIRA, M. M., 1987: Escavaçâo da mamoa 4 de Châ de Parada. Arqueologia, 16: 40-50.

KALB, P., 1987: Monumentos megaliticos entre Tejo e Douro. En El Megalitismo en la Peninsula lbérica: 95-109. Ministerio de Cultura, Madrid.

LOPEz Cuevillas, F., 1973: A edade megalitica, en R. Otero Pedrayo (ed.), Historia de Galicia, t. I., Buenos Aires, Ediciones Galicia: 43122.

Patino Gomez, R., 1984: Excavación de la mámoa 1 de Chan de Cruz (Vilaboa). Pontevedra Arqueológica, 1: 45-68.

Patino Gomez, R., 1984: Excavación de la mámoa n. ${ }^{\circ} 1$ de As Rozas (Campo Lameiro). Pontevedra Arqueológica, 1: 45-68. 
Pearson, G. W., Pllcher, J. R., Baille, M. G., Corbett, D. M. y Qua, F., 1986: High-Precision 14C Measurement of Irish Oaks to Show the Natural 14C Variations from AD 1840-5210 BC. Radiocarbon, 28: 911-934.

Peña Santos, A., 1985-86: La mámoa de As Pereiras (Mos, Pontevedra). Pontevedra Arqueológica, 2: 7-12.

Rodriguez Casal, A. A., 1979: O Megalitismo na Galiza. A sua problemática e o estado actual de investigación. En $1{ }^{\text {a }}$ Mesa-Redonda sobre o Neolitico e o Calcolitico em Portugal, Oporto: 103-115.

Rodriguez CASAL, A. A., 1983: Megalitico. Gran Enciclopedia Gallega 20: 215-220.

SILVA, E. J. L., 1984: O núcleo megalitico da Abogalheira e uma detaçäo pelo C-14. Revista de História, 1: 9-50.

VARIOS AUtORES, 1979: Prehistoria e Arqueoloxía de Galicia. Estado da cuestión. Lugo, Imp. La Voz de la Verdad.

VÁzQUez VARELA, J. M., 1979: El horizonte de Rechaba: Una nueva fase de la cultura megalitica del noroeste peninsular. Boletín Auriense, 9: 9-26. 